EPJ Web of Conferences 75, 05003 (2014)

DOI: 10.1051/epjconf/20147505003

(C) Owned by the authors, published by EDP Sciences, 2014

\title{
Focused Kerr measurements on patterned arrays of exchange biased square dots
}

\author{
G. Vinai ${ }^{1,2}$, J. Moritz ${ }^{1}$, G. Gaudin ${ }^{1}$, J. Vogel ${ }^{3}$, I.L. Prejbeanu ${ }^{1}$ and B. Dieny ${ }^{1}$ \\ ${ }^{1}$ SPINTEC, CEA/CNRS/UJF/Grenoble-INP; CEA/INAC, 17, rue des Martyrs, 38054, Grenoble, France \\ ${ }^{2}$ Crocus Technology, 4 Robert Schuman, 38025 Grenoble, France \\ ${ }^{3}$ Institut Néel, CNRS and UJF, BP166, 38042 Grenoble, France
}

\begin{abstract}
Microstructural effects on the antiferromagnetic layer were investigated on $\mathrm{IrMn} / \mathrm{Co}$ exchange biased square dots. IrMn grain size and distributions were tuned by changing $\mathrm{Cu}$ buffer layer or IrMn thicknesses. Lateral dimensions from 200 to $50 \mathrm{~nm}$ were varied. Exchange bias $\left(H_{e x}\right)$ variability was analysed through focused Kerr measurements on small groups of dots. Patterned samples presented average exchange bias values following the trends and values of full sheet samples. Concerning the dot to dot behaviour, it resulted that IrMn microstructure variations have minor effects on $H_{e x}$ variability, because no particular trend is observed as a function of grain size and distribution. The variability is attributed to geometry variation and Co intrinsic variability.
\end{abstract}

\section{Introduction}

For more than twenty years, the interfacial exchange coupling between a ferromagnetic (F) and an antiferromagnetic (AF) layer, known as exchange bias [1], has been exploited in various technological applications like spin valves [2] or Magnetic Random Access Memories (MRAM) [3].

Among the possible technologies for MRAM systems, Thermally Assisted MRAM (TA-MRAM) exploits exchange bias in both reference and storage layer [4]. In order to guarantee reproducibility and reliability, the variability of exchange bias properties from one memory point to another has to be minimized.

In this article, a microstructural study of the IrMn grain size distribution is presented for different buffer layer conditions and IrMn thicknesses through Atomic Force Microscopy (AFM) measurements. Samples were then patterned on square dots of different lateral sizes to analyse the effects of scalability and IrMn grain populations. It resulted that the effects of IrMn grain microstructure are secondary because covered by the intrinsic variability of the Co layer.

\section{Methodology of the study}

In exchange biased systems, the microstructure properties of both $\mathrm{AF}$ and $\mathrm{F}$ layers are an important parameter in the determination of the quality of the interface coupling and have important consequences on the thermal stability of the bilayer. Among these properties, the grain size of the
AF layer is important on sputtered systems. It has been observed $[5,6]$ that on polycrystalline systems the exchange bias intensity is proportional to the density of uncompensated $\mathrm{AF}$ spins at the interface. If a compensated AF spin structure is taken into account (as it is the case of IrMn [7]), uncompensated spins appear mainly at the grain boundaries. As a result, their density is inversely proportional to the AF grain diameter. The influence of the AF grain dimension has to be considered in combination with the thermal stability of the AF grain, which directly depends on its volume [8].

In IrMn polycrystalline thin layers, an increase of the AF thickness causes an enhancement of the average grain lateral size and a broadening of its distribution [9]. It is also possible to tailor $\mathrm{AF}$ grain population by changing the sputtering rate [10], the process pressure $[11]$, the annealing temperature $[12,13]$ or the buffering layer [12-15]. In this study, IrMn and buffering layer thicknesses were varied, without modifying the other parameters.

Concerning the grain measurement, different techniques are possible, including rocking curves of x-ray diffraction measurements $[12,15]$, Transmission Electron Microscopy grid scans [11] and AFM measurements [14, 16]. We preferred the last method because it is the only one that allows considering grain size distributions deposited on a $\mathrm{Si} / \mathrm{SiO}_{2}$ substrate. Grain measurements are intrinsically not absolute [17], but allow to have a relative comparison between samples. 


\section{Experimental procedure}

\subsection{Microstructural analysis}

The buffer layer series was composed by the following stack (thicknesses in $\mathrm{nm}$ ): $\mathrm{Ta}_{3} / \mathrm{Cu}_{\mathrm{x}} / \mathrm{IrMn}_{12} / \mathrm{Co}_{5} / \mathrm{Pt}_{2}$, with $\mathrm{x}$ ranging from 1 to $12 \mathrm{~nm}$, whereas the IrMn series was: $\mathrm{Ta}_{3} / \mathrm{Cu}_{2} / \mathrm{IrMn}_{\mathrm{x}} / \mathrm{Co}_{5} / \mathrm{Pt}_{2}$, with $\mathrm{X}$ ranging from 1 to $12 \mathrm{~nm}$. All samples were annealed at $473 \mathrm{~K}\left(200^{\circ} \mathrm{C}\right)$ for $30 \mathrm{~min}$ under a planar setting field of 2000Oe.

X-ray diffractometry measurements showed how the (111) fcc structure of the $\mathrm{Cu}$ layer allowed a good polycrystalline growth of the (111) fcc IrMn layer (not shown here).

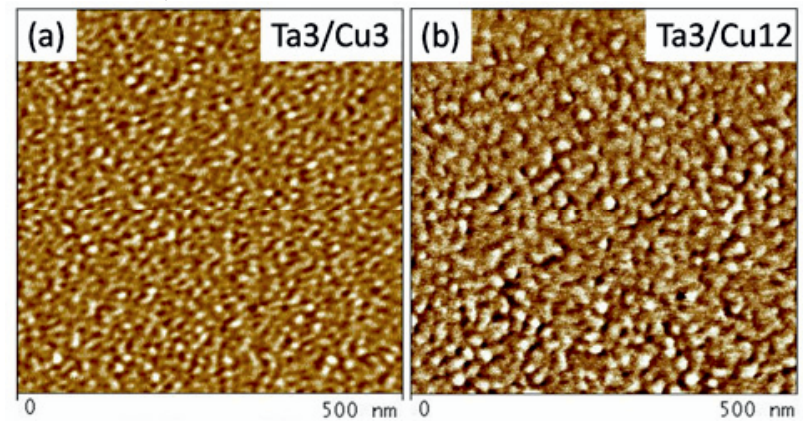

Fig. 1. AFM measurement for two different buffer layers

Figure 1 shows two examples of AFM measurements for different buffer layers. The topographic images, with a $\mathrm{z}$-scale of few $\mathrm{nm}$, allow distinguishing the granular structure of the grains, with white and dark contrasts. On each image, over 200 grains were circled and measured, giving a good statistics of the grain size distribution.

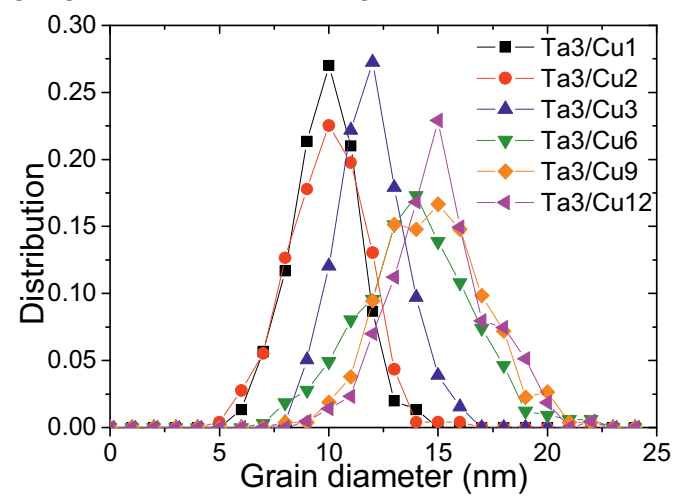

Fig. 2. Normalized frequency distribution for buffer layer series

Concerning the buffer layer series, figure 2 shows that, with the increase of the buffer thickness, the average grain diameter increases too in a monotonic way. Concerning the distribution width, higher grain size implies larger grain distribution. It can be observed comparing the AFM measurements of figure 1a and figure $1 \mathrm{~b}$. For thin $\mathrm{Cu}$ layer, small grains cover quite homogeneously the scanned surface, whereas for thicker $\mathrm{Cu}$ the variability of grains, among the large one and considering the presence of smaller grains in-between them, leads to a broader distribution.

Figure 3 shows the normalized frequency distributions for different IrMn thicknesses. The sputtered systems present an increase of the grain lateral size as the
IrMn thickness increases, together with a broadening of the distribution. This confirms the tendency observed from the buffer series in figure 3 (the larger the grains, the wider the distribution). Moreover, it is in accordance with the results obtained in the literature $[8,9]$, which showed how an increase of the IrMn thickness leads to an increase of the grain volume and a broadening of the distribution. The range of measured grain volumes fits well with those presented in the cited studies, thus confirming the validity of the measuring method.

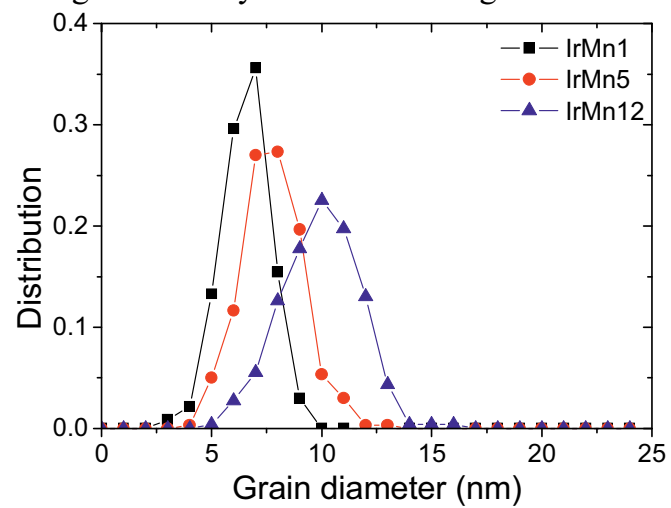

Fig. 3. Normalized frequency distribution for IrMn thicknesses

However, in our study the interest is not particularly focused on the volumetric properties of the grains, but on their lateral size. The aim of the study on patterned dots is to study the effect of the AF grain distribution on exchange bias variability: in the case of large grains with broad distribution, different dots may present very different IrMn grain populations, whereas for small and homogeneous grains the IrMn microstructure will not be very different from one dot to another. The goal is thus to reduce the grain lateral size so that the average lateral size becomes much smaller than the dot size in an MRAM system.

\subsection{Patterned systems}

The samples were then patterned by electron beam lithography and ion beam etching. As a result, only the patterned $\mathrm{F}$ dots contribute to the magnetic signal, the surface between dots being nonmagnetic. Three different geometries were processed: square dots $200 * 200 \mathrm{~nm}^{2}$ with an edge to edge spacing of $200 \mathrm{~nm}$ and square dots of $100 * 100 \mathrm{~nm}^{2}$ and $50 * 50 \mathrm{~nm}^{2}$ with an edge to edge spacing of $100 \mathrm{~nm}$. It can be considered from earlier studies that the dots are weakly interacting from a magnetostatic point of view [18].

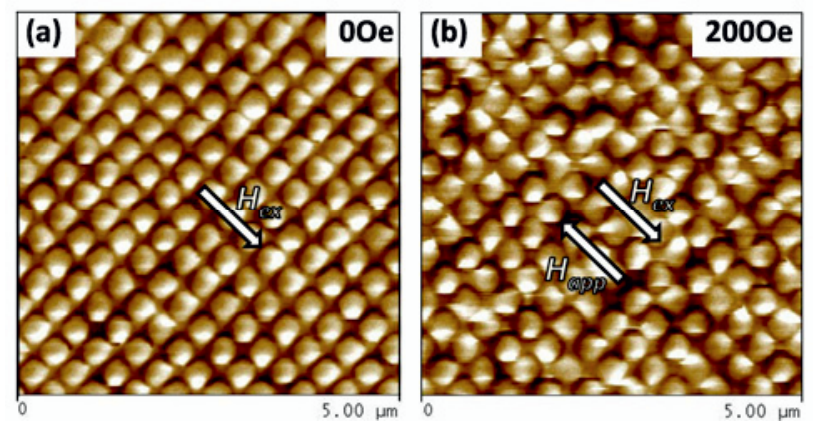

Fig. 4. MFM image on $200 \mathrm{~nm}$ square dots for $\mathrm{IrMn}_{3.5} / \mathrm{Co}_{5}$ sample at remanence (a) and under in-situ applied field (b) 
First, the micromagnetic configuration and reversal mechanism were analysed by Magnetic Force Microscope (MFM) scans with an in-situ applied field, with the following method:

- Saturation of the sample with an external field of 20000e along the setting field direction;

- MFM image at remanence (figure 4a);

- Application of an in-situ planar field along the easy axis, in the direction opposite to the setting field (figure $4 \mathrm{~b})$. The applied field was increased up to $40 \mathrm{mT}$ and then decreased down to $5 \mathrm{mT}$, to reproduce a half hysteresis loop. MFM images were performed at different applied fields.

- MFM image at remanence.

With a Co thickness of $5 \mathrm{~nm}$ for both buffer and IrMn thickness series, all samples presented a dipolar contrast at remanence, corresponding to a single domain state. Magnetization reversal takes place through in-plane coherent reversal. From figure $4 \mathrm{~b}$ it can be noticed that the different dots do not reverse at the same field and along the same rotation angle, giving a first qualitative picture of the switching field distribution present in the dots. This result is in accordance with a similar system measured in [19].

In order to have a more qualitative evaluation of the variability, fifty focused measurements were performed on each sample for all dot dimensions along the easy axis. The instrumentation consisted in a longitudinal focalized-MOKE. A He-Ne laser (wavelength $632 \mathrm{~nm}$ ) beam of $7 \mathrm{~mW}$ power was polarized and focused with a system of lenses. A 100x focalization lens focused the laser beam to a spot of around $600 \mathrm{~nm}$ diameter. This allows measuring about 2-3 dots for the $200 \mathrm{~nm}$ dot size, 9-10 dots for the $100 \mathrm{~nm}$ dot size and $14-15$ for the $50 \mathrm{~nm}$ cases. The reflected signal was separated by a Wollaston prism into two beams with orthogonal polarizations and then analysed by two photodiodes, allowing determining the magnetic signal of the sample. Coherently with the MFM scans, all loops showed a shifted single loop and variability in coercivity and loop shift (see figure 5) for all the considered lateral dimensions. On the other hand, full sheet samples presented negligible variations from one focused measurement to another, not overpassing the 10Oe.

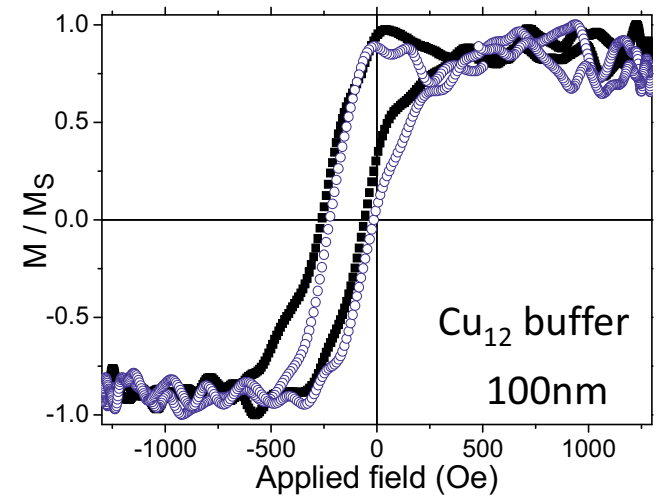

Fig. 5. Comparison between two focused measurements on two different zones in black and blue for $100 \mathrm{~nm} \mathrm{Cu}_{12} / \mathrm{IrMn}_{12} / \mathrm{Co}_{5}$ square dots

From the focalized measurements, exchange bias average value were calculated and compared between full sheet and patterned samples. The resulting curves for the two series are plotted on figure 6.
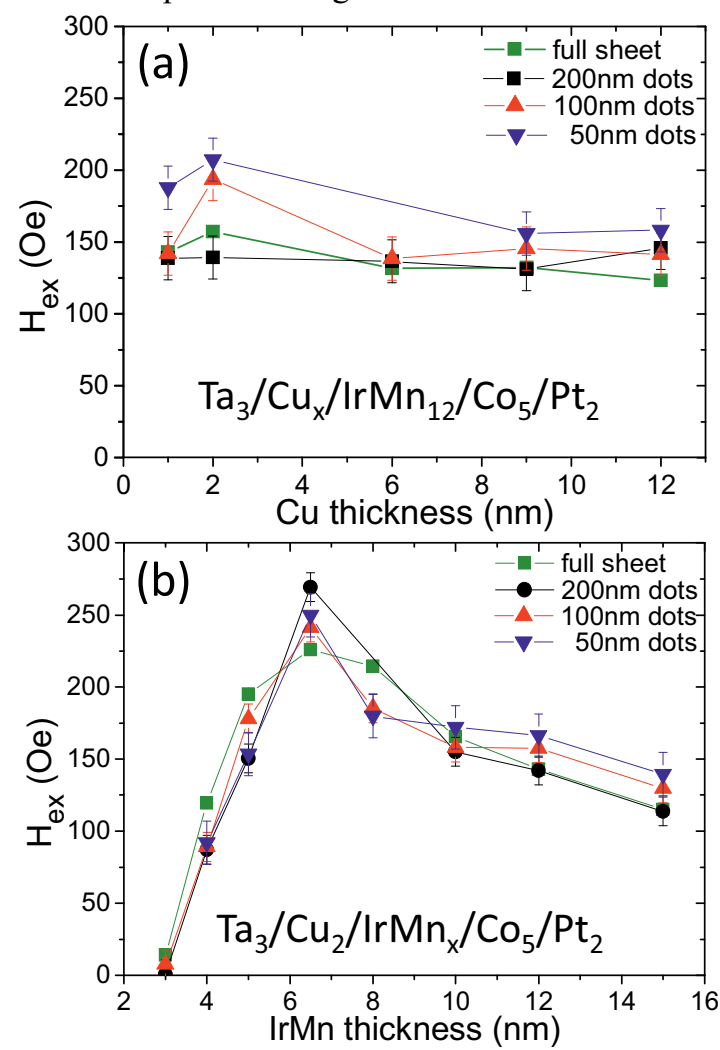

Fig. 6. Average exchange bias values for full sheet and patterned samples as a function of buffer thickness (a) and IrMn thickness (b)

Considering the buffer series of figure $6 \mathrm{a}$, no particular trend is observed. The influence on full sheet samples is quite limited, giving a weak exchange bias improvement for $2 \mathrm{~nm} \mathrm{Cu}$ buffer layer. Patterned dots have an average $H_{e x}$ around the value of the full sheet samples or slightly larger for the $50 \mathrm{~nm}$ dots case. It has to be underlined, however, how the differences in exchange due to the $\mathrm{Cu}$ thicknesses are already quite small in full sheet samples, and the incertitude of the focused measurements covers this variation.

Regarding the IrMn series, figure $6 \mathrm{~b}$ shows how the trend of the $H_{e x}$ curve does not change from patterned to full sheet samples. This means that in the selected dot geometry and lateral size range, $\mathrm{IrMn} / \mathrm{Co}$ dots average exchange bias behaviour does not deviate from the full sheet one. Comparing the result with the one obtained in [20], the phenomenon of $H_{e x}$ reduction at low IrMn thickness and the following enhancement on dots compared to continuous layer is much less pronounced. This may be due to the different composition of the magnetic stack ( $\mathrm{IrMn} / \mathrm{Co}$ instead of $\mathrm{NiFe} / \mathrm{IrMn})$, despite the similarity in the dot geometry.

The first conclusion from the averaged behaviour is that changes in the IrMn microstructure (grain volume and lateral size distributions) do not imply differences in the average behaviour between patterned dots and full sheet samples. This is valuable for a lateral size range from 200 to $50 \mathrm{~nm}$, where no scalability effects are observed. 

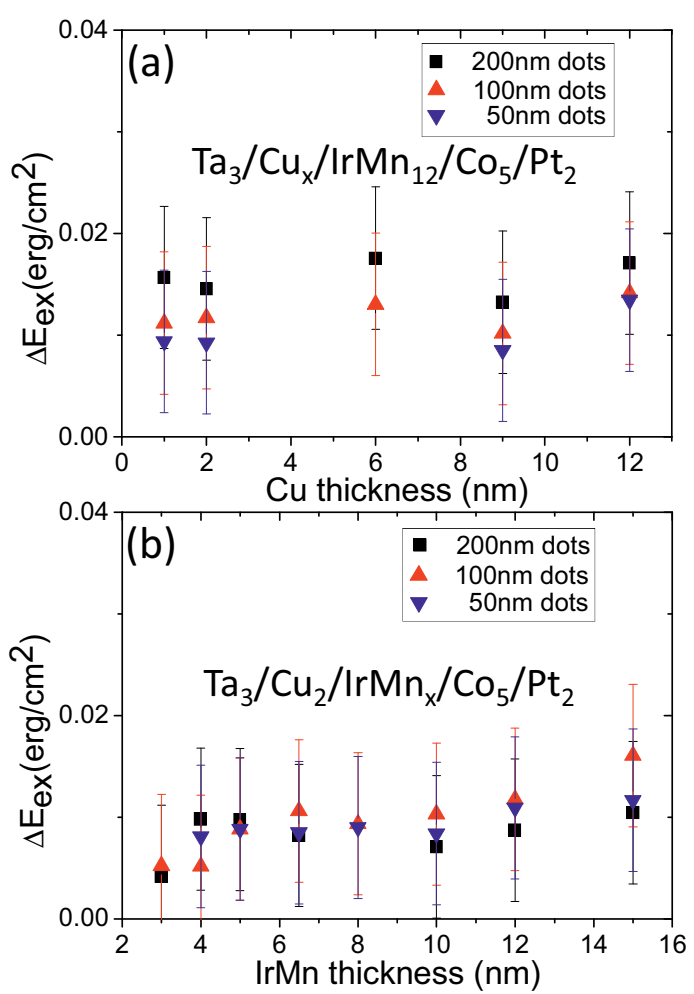

Fig. 7. Exchange bias variability for patterned dots of buffer thickness (a) and IrMn thickness (b) series

Is there any effect on the dot to dot variability? To evaluate it, we calculated the variation of the exchange energy $E_{e x}$, defined as the product between the exchange bias field, the Co thickness and the Co magnetization saturation $\left(-1400 \mathrm{emu} / \mathrm{cm}^{3}\right)$ [19]. Figure 7 shows the exchange bias variability $\Delta E_{e x}$ for the two cases.

In figure $7 \mathrm{a}$ and $7 \mathrm{~b}$, both exchange energy variation values are included in an envelope. In figure $7 \mathrm{~b}$, the slight increase as a function of $t_{I r M n}$ is not large enough to justify a direct implication of the IrMn microstructure, in particular if compared to the $\Delta E_{e x}$ trend observed between thin and thick Co layers in [19]. Similarly it can be said for the buffer layer case, where no clear trend can be observed as a function of IrMn grain size. Concerning the scalability effect, the values of exchange energy variability do not differ particularly as a function of the lateral size. This information has to be treated carefully because of the different number of dots averaged per measurements. Smaller dots do not show a marked variability increase compared to larger ones, as it happened for thick Co layer [19].

\section{Conclusions}

Focused Kerr measurements showed that the microstructural properties of $\mathrm{IrMn}$ in $\mathrm{IrMn} / \mathrm{Co}$ square dots have a minor effect on exchange energy variability on the considered dot dimension and magnetic stacks. The hysteresis loop variations are ascribed to small geometry changes and micromagnetic effects on the Co layer.

For technological applications, these results suggest that particular attention has to be put on the $\mathrm{F}$ layer more than on the AF one. To further reduce the variability, softer F materials like NiFe or synthetic antiferromagnets may help reducing the exchange energy variability.

\section{Acknowledgements}

We would like to thank Sébastien Bandiera for the helpful discussions.

This work was partially funded by the European Commission through the Adv ERC project HYMAGINE $n^{\circ} 246942$.

\section{References}

1. J. Nogués and I.K. Shuller, J. Magn. Magn. Mater. 192, 203 (1999)

2. B. Dieny, V.S. Speriosu, S.S.P. Parkin, B.A. Gurney, Wilhoit D.R. and D. Mauri, Phys. Rev. B 43, 1297 (1991)

3. I.L. Prejbeanu, S. Bandiera, J. Alvarez-Hérault, R.C. Sousa, B. Dieny and J.P. Nozières, J. Phys. D: Appl. Phys. 46, 074002 (2013)

4. I.L. Prejbeanu, M. Kerekes, R.C. Sousa, H. Sibuet, O. Redon, B. Dieny and J.P. Nozières, J. Phys.: Condens. Matter 19, 165218 (2007)

5. K. Takano, R.H. Kodama, A.E. Berkowitz, W. Cao and G. Thomas, Phys. Rev. Lett. 79, 1130 (1997)

6. D. Martien, K. Takano, A.E. Berkowitz and D.J. Smith, Appl. Phys. Lett. 74, 1314 (1999)

7. I. Tomeno, I.N. Fuke, H. Iwasaki, M. Sahashi and Y. Tsunoda, J. Appl. Phys. 86, 3853 (1999)

8. K. O'Grady, L.E. Fernandez-Outon and G. VallejoFernandez, J. Magn. Magn. Mater. 322, 883 (2010)

9. G. Vallejo-Fernandez, L.E. Fernandez-Outon and K. O’Grady, J. Phys. D: Appl. Phys. 41, 112001 (2008)

10. S. Manzoor, M. Vopsaroiu, G. Vallejo-Fernandez and K. O’Grady, J. Appl. Phys. 97, 10K118 (2005)

11. M. Vopsaroiu, M. J. Thwaites, S. Rand, P.J. Grundy and O'Grady, IEEE Trans. Magn. 40, 2443 (2004)

12. J. Kanak, T. Stobicki, O. Schebaum, G. Reiss and H. Brückl, Phys. Stat. Sol (b) 243, 197 (2006)

13. M. Xu, Z. Lu, T. Yang, C. Liu, Z. Mai and W. Lai, J. Appl. Phys. 92, 2052 (2002)

14. M.H. Li, J.W. Cai, G.H. Yu, H.W. Jiang, W.Y. Lai and F.W. Zhu, Mater. Sci. Eng. B 90, 296 (2002)

15. M. Pakala, Y. Huai, G. Anderson and L. Miloslavsky, J. Appl. Phys. 87, 6653 (2000)

16. M. Fecioru-Morariu, S.R. Ali, C. Papusoi, M. Sperlich and G. Güntherodt, Phys. Rev. Lett. 99, 097206 (2007)

17. D.T. Carpenter, J.R. Codner, K. Barmak and J.M. Rickman, Mater. Lett 41, 296 (1999)

18. M. Natali, I.L Prejbeanu, A. Lebib, L.D. Buda, K. Ounadjela and Y. Chen, Phys. Rev. Lett. 88, 157203 (2002)

19. G. Vinai, J. Moritz, G. Gaudin, J. Vogel, M. Bonfim, F. Lançon, I.L. Prejbeanu, K Mackay and B. Dieny, J. Phys. D: Appl. Phys. 46, 345308 (2013)

20. V. Baltz, J. Sort, S. Landis, B. Rodmacq and B. Dieny, Phys. Rev. Lett. 94, 117201 (2005) 